\title{
The Death Lines of Radio Pulsars for Dipolar and Asymmetric Magnetic Fields
}

\author{
E. M. Kantor and A. I. Tsygan \\ Ioffe Physicotechnical Institute, Russian Academy of Sciences, \\ Politekhnicheskaya ul. 26, St. Petersburg, 194021 Russia \\ Received March 23, 2004; in final form, July 15, 2004
}

\begin{abstract}
The effect of curvature of open magnetic-field tubes on the death lines of radio pulsars is studied. The solution is obtained in the framework of a Goldreich-Julian model for both dipolar and asymmetric magnetic fields. The tube-axis curvature can shift the death line appreciably toward either longer or shorter periods. If the field is dipolar and gamma rays are generated by the inverse Compton effect, the formation of secondary plasma is more efficient near the death line. In the case of an asymmetric magnetic field, the generation of radio emission beyond the tube of open field lines is possible. (C) 2004 MAIK "Nauka/Interperiodica".
\end{abstract}

\section{INTRODUCTION}

The death line of a radio pulsar is the curve in the magnetic-field-period plane separating the regions where radio emission can and cannot be generated. The mechanism for the radio emission is as follows $[1,2]$. Electrons are accelerated by the electric field along open magnetic field lines emanating from the polar regions of the pulsar (we shall consider the case of an acute angle between the angular-velocity and magnetic-moment vectors). Next, these electrons produce gamma rays, which, in turn, generate secondary plasma (i.e., electron-positron pairs) due to the presence of a transverse component of the magnetic field. When the primary high-energy particles pass through the secondary plasma, they generate oscillations that result in radio emission. It is commonly believed that a pulsar will no longer emit at radio frequencies if a sufficient amount of secondary plasma cannot be formed.

In the present work, we shall calculate the death lines for an asymmetric magnetic field and compare these results with the case of a dipolar field. The death lines for a dipolar field have already been obtained by other authors (for example, in the recent works [3] and [4]). However, we calculate them again to show that our approximation method gives the same results for the dipolar field as a numerical approach [3]. In addition, we study the effect of stationary plasma in unfavorably bent field lines. When the field is dipolar and gamma rays are formed by the inverse Compton effect, the generation of secondary plasma is more efficient near the death line. In the case of an asymmetric magnetic field, radio emission can be generated beyond the tube of open field lines.
To calculate the death lines, we need information about the electric field accelerating the primary electrons.

In a regime with the free outflow of charges in a Goldreich-Julian model for a rotating neutron star possessing a magnetic field (a radio pulsar), the electric fields in the region of open magnetic field lines are determined by the relativistic effect of inertial reference-frame dragging. This was demonstrated for the case of a dipolar magnetic field by Muslimov and Tsygan [5, 6] and Beskin [7], and for an arbitrary axially symmetric magnetic field by Tsygan [8].

The electric field for the case of curved axes of the tubes of open field lines of a nondipolar magnetic field was calculated by Kantor and Tsygan [9].

We derived the death lines in analytic form. The emission spectra and energy distributions of the secondary plasma were not taken into account, and all calculations were carried out for the characteristic energies.

\section{DEATH LINES FOR A DIPOLAR FIELD WITHOUT STATIONARY PLASMA IN THE REGION OF OPEN FIELD LINES}

As is known, the electrostatic potential (in a coordinate system rotating with a star) in the region of open dipolar magnetic field lines at distances much less than the light-cylinder radius is $[5,6]$

$$
\begin{gathered}
\Phi=\frac{1}{2} \kappa \Theta_{0}^{2}\left(\frac{\Omega a}{c}\right) B_{0} a\left(1-\xi^{2}\right)\left(1-\frac{1}{\eta^{3}}\right) \cos \chi \\
\cos \chi \neq 0 .
\end{gathered}
$$


Here, $\kappa=\left(r_{g} / a\right)\left(I / M a^{2}\right)$, where $I$ is the moment of inertia of the star, $r_{g}=(2 G M) / c^{2}$ is the gravitational radius of a neutron star with mass $M$, and $a$ is its radius. The parameter $\kappa$ describes the effect of inertial-frame dragging (described by the metrictensor component $g_{03}$ ) on the electric field near the star; its characteristic value is equal to 0.15 . Further, $\Omega$ is the angular velocity of the star, $B_{0}$ is the amplitude of the magnetic field at the magnetic pole of the star, $\Theta_{0}=\sqrt{\Omega a / c}$ is the angular radius of a tube of open field lines at the stellar surface, $\xi$ is the distance from the tube axis normalized to $\Theta_{0}, \eta$ is the distance from the stellar center normalized to its radius, and $\chi$ is the angle between the magnetic moment and angular velocity of the star. We shall not take into account the Schwarzschild terms $g_{00}$ and $g_{11}$ in the metric tensor describing the gravitational field near the rotating neutron star. Therefore, an accelerated electron will acquire the Lorentz factor

$$
\gamma=\frac{e \Phi_{d i p}}{m c^{2}}=10^{6} P^{-2} B_{12}\left(1-\frac{1}{\eta^{3}}\right) \cos \chi,
$$

where $P$ is the period of the radio pulsar in seconds and $\xi$ was taken to be 0.5 , since this corresponds to a line with a not very small potential and curvature simultaneously.

The free path of a photon in the magnetic field is determined in the standard way $[1,10]$. The photons are emitted by electrons along the magnetic field lines within an angle of about $1 / \gamma$. Before a pair is born in a magnetic field with radius of curvature $\rho$, the photon traverses the path

$$
S_{p h}=\frac{0.2 m c^{2} \rho}{\hbar \omega} \frac{B_{c}}{B} .
$$

This expression can be obtained if the integral of the photon absorption coefficient in the magnetic field along the photon trajectory is assumed to be unity. The main contribution to the integral is produced by the final part of the trajectory (where the pair is produced). Therefore, the magnetic field appearing in this formula is the field in the place where the pair is formed. Our estimate is valid for $B<10^{13} \mathrm{G}$, because it does not take into account the processes considered in [11], such as deflection of the gamma ray by the magnetic field, the formation of a pair in a bound state, and the decay of one of the states of the gamma ray into two photons.

For a dipolar field, the radius of curvature $\rho$ at $\xi=0.5$ is equal to $2.2 \times 10^{8} \mathrm{P}^{1 / 2} \mathrm{~cm}$. Therefore,

$$
S_{p h} \approx 2 \times 10^{9} \frac{m c^{2}}{\omega \hbar} \frac{P^{1 / 2}}{B_{12}} \mathrm{~cm} .
$$

The photons emitted by the primary electrons will have the following energies. Magnetic curvature radiation results in gamma rays with characteristic energies $E_{p h}=(3 / 2)\left(\gamma^{3} \hbar c / \rho\right)$. The inverse Compton scattering of thermal photons on electrons with Lorentz factors $\gamma>m c^{2} / 2 k T$ results in gamma rays with energy $E_{p h}=m c^{2} \gamma$. When $\gamma<m c^{2} / 2 k T$, gamma rays with energy $E_{p h}=2 k T \gamma^{2}$ are generated [12].

The condition for the creation of a pair is $[2,10]$

$$
B_{\perp}=B_{c} \frac{0.2 m c^{2}}{\hbar \omega} .
$$

Here, $B_{\perp}$ is the magnetic-field component perpendicular to the direction of propagation of the gamma ray.

As the gamma ray moves away from the place it is emitted, the angle between the magnetic field and the direction of propagation increases, but the intensity of the field decreases. The transverse component of the field will be maximum at the point $\eta_{0}=1.5 \eta$, where $\eta$ is the distance from the stellar center to the place where the gamma ray is emitted.

The condition $\eta_{0}=1.5 \eta$ can be rewritten

$$
S_{p h}=\frac{10^{15}(1.5 \eta)^{3} P^{1 / 2}}{B_{12} \omega \hbar(\eta)}=\frac{\eta}{2} 10^{6},
$$

where $B_{12}$ is the magnetic-field intensity at the stellar surface, and $\omega \hbar(\eta)$ is the energy of a photon produced at a distance $\eta$ from the stellar center.

In the case of inverse Compton scattering by energetic electrons, we have

$$
\begin{gathered}
S_{p h}=\frac{10^{15}(1.5 \eta)^{3} P^{1 / 2}}{B_{12} 5 \times 10^{5} \times 10^{6} P^{-2} B_{12} \cos \chi\left(1-\frac{1}{\eta^{3}}\right)} \\
=\frac{\eta}{2} 10^{6} .
\end{gathered}
$$

After some manipulation of this equation, we obtain $\eta^{2} /\left(1-1 / \eta^{3}\right)=0.74 \times 10^{2} B_{12}^{2} P^{-2.5} \cos \chi$. The furthest right line in the $B-P$ plane corresponds to the minimum of the function $\eta^{2} /\left(1-1 / \eta^{3}\right)$, i.e., $\eta \approx$ 1.35. Therefore, the death line is described by the expression

$$
P=3.6 B_{12}^{0.8}(\cos \chi)^{0.4} \text {. }
$$

\section{MULTIPLICATION FACTOR FOR THE SECONDARY PLASMA IN A DIPOLAR FIELD WITHOUT STATIONARY PLASMA IN THE REGION OF OPEN FIELD LINES}

To determine this multiplication factor, we need to know how many gamma rays are produced by a single primary electron and how many pairs are formed by a single gamma ray. It is obvious that a cascade cannot 
develop near the death line, and that one gamma ray cannot produce more than one pair.

The number of gamma rays produced is determined by the integrated probability of gamma ray formation. Gamma rays born very close to the stellar surface cannot form pairs because their energy is insufficient, whereas gamma rays born at very large heights cannot form pairs because the magnetic field decreases with distance from the star. The corresponding boundaries are defined by the condition that the distance from the stellar center to the point where the pair is formed is a factor of 1.5 greater than the distance from the stellar center to the point where the gamma ray is formed. From here on, we shall consider the inverse Compton scattering of thermal photons on fast electrons $\left(\gamma>m c^{2} / 2 k T\right)$, when the gamma ray carries away all the energy of the electron. For this condition to be satisfied, it is necessary that $T_{5} P^{0.5} / B_{12}>1.5$. In the conditions typical for radio pulsars, the probability of photon scattering by an electron somewhere along its entire path is much less than unity.

Photons produced at a resonant cross-section will contribute to the formation of pairs if [12]

$$
\gamma_{\text {res }}=10^{3} \frac{B_{12}}{T_{5}}>\frac{1.4 \times 10^{4} P^{0.5}}{B_{12}},
$$

in other words,

$$
\frac{B_{12}^{2}}{T_{5} P^{0.5}}>14
$$

If this requirement is not satisfied, the number of photons scattered by one electron along the path from $r_{1}$ to $r_{2}$ is

$$
N_{\gamma}=\int_{r_{1}}^{r_{2}} \frac{\frac{\sigma_{T} \sigma T^{4}}{c}\left(\frac{m c^{2}}{k T}\right)^{2} \ln \left(\frac{2 \gamma k T}{m c^{2}}\right) d L}{m c^{2} \gamma},
$$

where $\ln \left(2 \gamma k T / m c^{2}\right)$ is a quantity of the order of unity, and $r_{1}$ and $r_{2}$ are the upper and lower boundaries for the generation of pair-forming photons. Therefore, we obtain

$$
N_{\gamma}=\int_{r_{1}}^{r_{2}} \frac{T_{5}^{2}}{2.3 \times 10^{3} \gamma} d L
$$

Consequently, the number of gamma rays generated by one electron $N_{\gamma}$ when thermal emission is produced by the entire surface of the star is equal to

$$
N_{\gamma}=\frac{7 \times 10^{-4} T_{5}^{2} P^{2}}{B_{12} \cos \chi} .
$$

We can see that the multiplication factor increases near the death line (we assume here that one gamma ray creates one pair, and that the multiplication factor equals $N_{\gamma}$; the condition for the formation of a cascade will be discussed below). If the amplitude of the magnetic field is fixed, the concentration of the secondary plasma increases as $P$. As a result, the efficiency of radio emission should increase near the death line. The increase in the concentration will be stopped and replaced with a decrease when the height $\eta$ (the height above which photons created cannot produce pairs) approaches the value $\eta=2$ (the height at which the flux of thermal photons from the stellar surface is substantially attenuated). This takes place when $P=3.2 B_{12}^{4 / 5} \cos ^{2 / 5} \chi$; i.e., the true death line should be within the interval $P=$ $(3.2-3.6) B_{12}^{0.8} \cos ^{0.4} \chi$.

We have not taken into account screening of the field by the electron-positron plasma, since the multiplication factor was small (this is valid for moderate temperatures of the stellar surface $T_{5}<10$, where $\left.T_{5}=T / 10^{5} \mathrm{~K}\right)$.

Let us consider the case when thermal radiation is emitted only by a hot spot in the polar region. Secondary plasma is formed as long as gamma rays creating pairs are produced at heights $z_{0}<0.01 P^{-1 / 2}$, since the flux of thermal photons is considerably attenuated at heights exceeding the radius of the polar region.

Therefore, the death line will be given by

$$
P<1.35 B_{12}^{2 / 3}(\cos \chi)^{1 / 3} .
$$

The number of photons produced will be

$$
N_{\gamma}=\frac{1.9 \times 10^{-4} T_{5}^{2} P^{1 / 2}}{\cos ^{1 / 2} \chi} .
$$

Next, let us consider the case when the photons are generated by magnetic curvature radiation. A similar calculation of the death line gives

$$
P=0.16 B_{12}^{4 / 7} \cos ^{3 / 7} \chi .
$$

Let us determine the number of gamma rays emitted per electron that are able to create a pair. Since the generation of secondary plasma becomes efficient in the case of magnetic curvature radiation, screening of the electric field should be taken into account. We shall assume that the electric field is zero everywhere above the point where the first electron-positron pair was born.

The minimum pair-creation height (the height of the gap) in the case of $P>0.08 B_{12}^{8 / 17} \cos ^{6 / 17} \chi$ will be $h=7.5 P^{7 / 4} /\left(B_{12} \cos ^{3 / 4} \chi\right)$, and the Lorentz factor at this height reaches $\gamma(h)=2.3 \times 10^{7} P^{-1 / 4} \cos ^{1 / 4} \chi$. 
To obtain the corresponding expressions for the case $P<0.08 B_{12}^{8 / 17} \cos ^{6 / 17} \chi$, the effect of the secondary plasma on the electric field must be taken into account. We shall not consider this case.

A single electron generates the following number of photons that are able to form pairs:

$$
N_{\gamma} \approx \frac{10^{6} \gamma(h) \eta_{0}}{2 P^{1 / 2} c}
$$

where $\eta_{0}=1+z_{0}$, and $z_{0}$ is the height above which the photons can create pairs.

$$
\begin{aligned}
& \text { When } h>0.01 P^{-1 / 2} \text {, we find } \\
& \qquad N_{\gamma}=\frac{2.3 \times 10^{2} B_{12}^{1 / 2} \cos ^{5 / 8} \chi}{P^{13 / 8}} .
\end{aligned}
$$

Since the number of photons we have obtained is much greater than for the inverse Compton effect, it is reasonable to assume that the true death line should be located to the right of the one obtained above. Pairs will not be formed from electrons with the characteristic energy, but they will form from electrons in the high-energy tail of the spectrum, although the number of pairs produced will be decreased by a factor of $e^{E / E_{0}}$, where $E_{0}=(3 / 2)\left(\gamma^{3} \hbar c / \rho\right)$. If we take $E=$ $10 E_{0}$, the number of pair-forming gamma rays will be decreased by a factor of $2 \times 10^{4}$; i.e., this number will be comparable to that for the inverse Compton effect. The corresponding death line will be described by the expression

$$
P=0.22 B_{12}^{4 / 7} \cos ^{3 / 7} \chi,
$$

which agrees well with the results [4].

If a gamma ray possesses a sufficiently large energy, the electron-positron pair produced will have a large transverse momentum. The momentum of a particle across the magnetic field will be reduced by radiation, and the energy of the photons emitted could be sufficient to generate secondary pairs [13]. Therefore, a cascade can develop. However, this is impossible near the death line, and the multiplication factors will be equal to the number of gamma rays produced by a single electron.

Therefore, the multiplication factors for the dipolar field will be as follows. In the case of the magnetic curvature generation of gamma rays, the multiplication factor is

$$
k=\frac{2.3 \times 10^{2} B_{12}^{1 / 2} \cos ^{5 / 8} \chi}{P^{13 / 8}}
$$

if $P>0.08 B_{12}^{8 / 17} \cos ^{6 / 17} \chi$ (this is the condition for the absence of a cascade) and the spot under consideration is not very close to the death line.
In the case of inverse Compton scattering of thermal photons from the entire surface of the star, the multiplication factor is

$$
k=\frac{7 \times 10^{-4} T_{5}^{2} P^{2}}{B_{12} \cos \chi} .
$$

This is valid for the parameter ranges $0.75 B_{12}^{0.8} \times$ $\cos ^{0.4} \chi<P<3.2 B_{12}^{0.8} \cos ^{0.4} \chi, P>2.3 B_{12}^{2} / T_{5}^{2}$.

In the case of inverse Compton scattering of thermal photons from a hot spot in the polar region, the multiplication factor is

$$
k=\frac{1.9 \times 10^{-4} T_{5}^{2} P^{1 / 2}}{\cos ^{1 / 2} \chi} .
$$

This is valid not very close to the death line at $P>$ $2.3 B_{12}^{2} / T_{5}^{2}$ and $P>0.3 B_{12}^{2 / 3} \cos ^{1 / 3} \chi$.

\section{STATIONARY PLASMA IN THE REGION OF OPEN FIELD LINES}

Let us consider the electrostatic potential in the region of open magnetic field lines. As is known, there are favorable and unfavorable lines [15]. The potential varies monotonically along favorable lines up to the light cylinder, whereas there are potential wells on unfavorable lines.

The electrostatic potential in the case of a dipolar magnetic field takes the form [6]:

$$
\begin{gathered}
\Phi=\frac{1}{2} \Phi_{0} \kappa \Theta_{0}^{2}\left(1-\frac{1}{\eta^{3}}\right)\left(1-\xi^{2}\right) \cos \chi \\
+\frac{3}{8} \Phi_{0} \Theta_{0}^{3} H(1)\left(\frac{\Theta(\eta) H(\eta)}{\Theta_{0} H(1)}-1\right) \\
\quad \times \xi\left(1-\xi^{2}\right) \sin \chi \cos \phi,
\end{gathered}
$$

where the following notation has been used:

$$
\begin{gathered}
\Phi_{0}=(a \Omega / c) B_{0} a, \\
\Theta_{0}=\sqrt{\Omega a / c f(1)}, \\
\Theta(\eta)=\Theta_{0}\left(\eta \frac{f(1)}{f(\eta)}\right)^{1 / 2}, \\
f(\eta)=-3\left(\frac{\eta}{\varepsilon}\right)^{3}\left[\ln \left(1-\frac{\varepsilon}{\eta}\right)+\frac{\varepsilon}{\eta}\left(1+\frac{\varepsilon}{2 \eta}\right)\right], \\
\text { where } \varepsilon=r_{g} / a \approx 0.4, \\
H(\eta)=\frac{1}{\eta}\left(\varepsilon-\frac{\kappa}{\eta^{2}}\right) \\
+\left(1-\frac{3}{2} \frac{\varepsilon}{\eta}+\frac{1}{2} \frac{\kappa}{\eta^{3}}\right)\left\{f(\eta)\left(1-\frac{\varepsilon}{\eta}\right)\right\}^{-1},
\end{gathered}
$$

$\phi$ is the azimuth angle in a cylindrical coordinate system whose axis is directed along the tube.

ASTRONOMY REPORTS Vol.48 No. 122004 


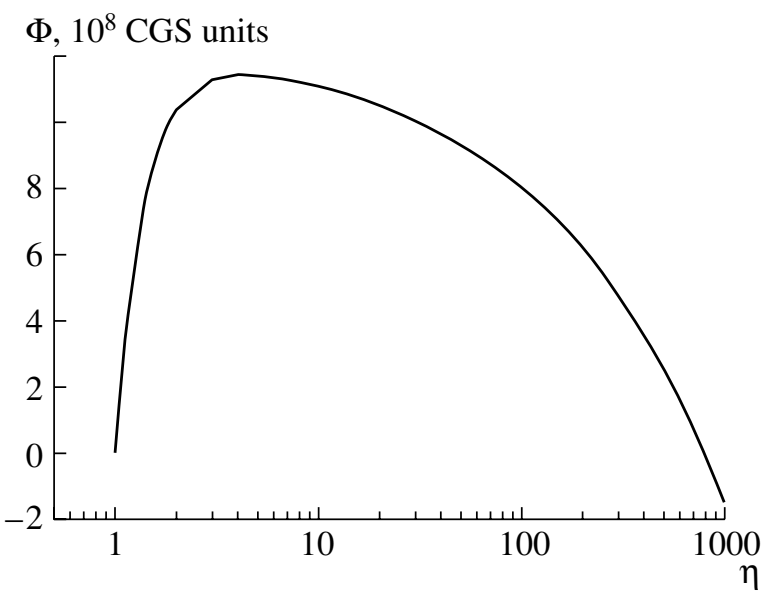

Fig. 1. Behavior of the potential for $\xi=0.5, \chi=45^{\circ}$, $\cos \phi=-1, P=1, B_{12}=1$.

At some angles $\phi$, the potential will be nonmonotonic; i.e., there will be potential wells (Fig. 1).

If the formation of electron-positron plasma does not proceed very actively, the charged particles will be concentrated in these wells and tend to smooth the potential. Therefore, the following situation will develop in unfavorably bent field lines. The potential is zero at the stellar surface. The next layer is filled with a stationary plasma, and the potential remains zero up to some point, where a potential with an opposite sign relative to the favorable lines appears, which varies monotonically. There is no electron flux from the stellar surface along these lines (we consider the case of an acute angle between the angular velocity of the star and its magnetic moment), but there can be an inverse flow of electrons from the light cylinder. The presence of this stationary plasma with zero potential should decrease the effective cross-section of the tube, and, consequently, the value of the accelerating potential.

If a considerable amount of plasma is formed (at small periods, when magnetic curvature radiation is efficient), the electric field can become zero everywhere above the place where the plasma is formed. Therefore, the potential will be monotonic, and there will be no decrease in the tube cross-section, or, consequently, in the potential. On the other hand, it is also possible that some part of the tube will be filled. We cannot definitely answer which of these cases actually takes place.

Let us consider the influence on the death line of decreasing the cross-section of the tube in which the electrons are efficiently accelerated.

To estimate the electrostatic potential, let us assume that the part of the tube where the electron acceleration takes place possesses a circular crosssection with a radius that is half the radius of the

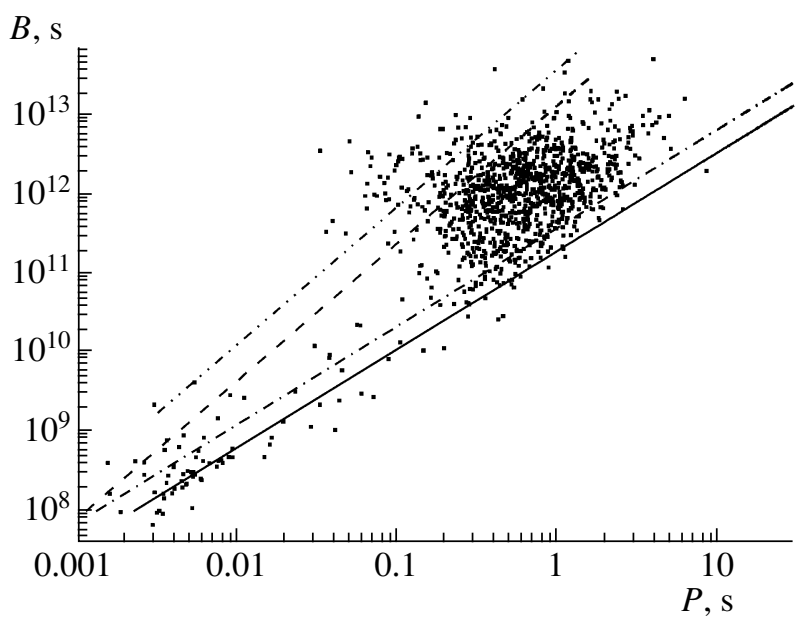

Fig. 2. Calculated death lines of radio pulsars for the magnetic curvature mechanism (dashed), the inverse Compton effect acting on thermal photons from the entire stellar surface with stationary plasma (dash-dot), the inverse Compton effect acting on thermal photons from the entire stellar surface without stationary plasma (solid), and the magnetic curvature mechanism with stationary plasma (dash-double-dot). The observational data (points in the $B-P$ plane) were taken from the unpublished ATNF Pulsar Catalog of Hobbs and Manchester (2003).

initial tube. Since the potential depends quadratically on the cross-sectional radius, the electron Lorentz factor should decrease by a factor of four. The death line corresponding to the inverse Compton effect acting on photons from the entire surface of the star will be $P=(1.8-2.1) B_{12}^{0.8} \cos ^{0.4} \chi$, that corresponding to the inverse Compton effect acting on photons from a hot spot will be $P=0.8 B_{12}^{2 / 3}(\cos \chi)^{1 / 3}$, and that corresponding to magnetic curvature radiation $P=$ $0.12 B_{12}^{4 / 7} \cos ^{3 / 7} \chi$. These death lines and the corresponding lines without filling taken into account are drawn in Figs. 2 and 3.

\section{DEATH LINES IN THE CASE OF BENT TUBES (AN ASYMMETRIC MAGNETIC FIELD)}

Let us describe the specific features of bent tubes.

The potential in an asymmetric tube can be expressed [9]

$$
\begin{gathered}
\Phi=\frac{\Omega F}{2 \pi c}\left(1-\xi^{2}\right) \\
\times\left\{\left[1-\kappa(1 / \eta)^{3}\right] \cos \chi+(\kappa-1) \cos \chi_{s}\right\},
\end{gathered}
$$

where $F$ is the magnetic flux through the tube, $\cos \chi_{s}$ is the cosine of the angle between the angular velocity and the magnetic field near the stellar surface, and $\cos \chi$ is the cosine of the angle between the angular 


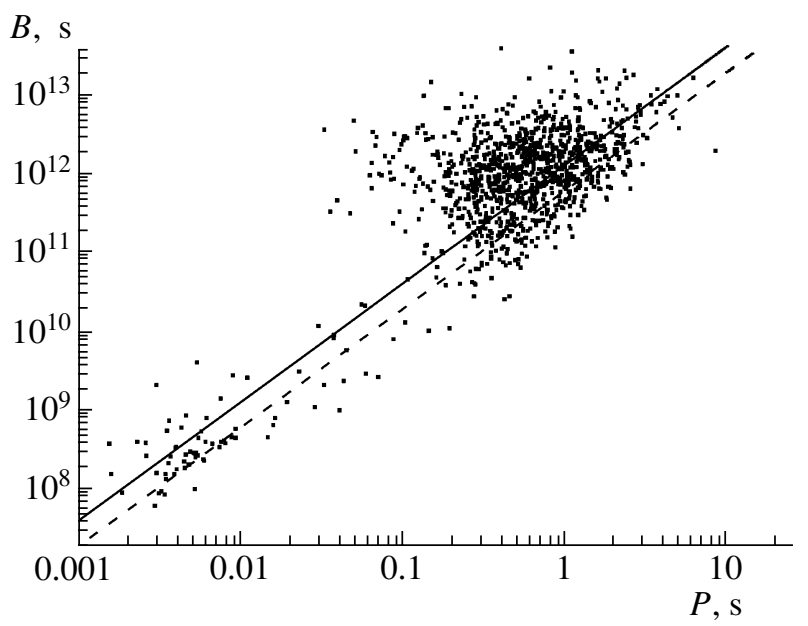

Fig. 3. Same as Fig. 2 for the inverse Compton effect acting on thermal photons from a hot spot in the polar region when stationary plasma is present (solid) and the inverse Compton effect acting on thermal photons from a hot spot in the polar region without stationary plasma (dashed)

velocity and the magnetic field at the point under consideration.

The following model was considered in our previous work [9]. A star with radius $a$ possesses magnetic moment $\mathbf{m}$, so that the field at its magnetic pole is $B_{0}=2 \mathrm{~m} / \mathrm{a}^{3}$. An additional dipole with magnetic moment $\mathbf{m}_{1} \perp \mathbf{m}$ is located at a depth $a \Delta(\Delta \approx 0.1)$ near the pole of the neutron star, so that its field at the star's pole is equal to $B_{1}=m_{1} / a^{3} \Delta^{3}$. Assuming that $B_{0}=B_{1}$ and a favorable axis inclination and tube curvature, we can obtain an increase by a factor of five in the potential and by a factor of 20 in the curvature, compared to the dipolar case. The corresponding potential wells will be shallower, and they will not be filled with stationary charge to the zero potential level. However, we can still describe the filling as a halving of the effective cross-section of the tube.

One characteristic feature of tubes with bent axes is that electron-positron pairs can be produced both inside and outside the tube (in the region of closed field lines). The birth of pairs in the region of closed field lines is possible because, if a photon does not have too large an energy, its path before the formation of a pair can be large enough for it to leave the tube. This path length must satisfy the condition $L>$ $\sqrt{\rho \Theta a \eta}$ (where $\rho$ is the radius of curvature of the tube, and $\Theta a \eta$ is the tube radius). After traversing the entire magnetosphere, pairs born near tubes in the region of closed field lines will enter the region of the opposite magnetic pole. We might expect that they would heat a spot near this pole, but this heating was found to be negligible, because pairs produced beyond the tube have a fairly small Lorentz factor (about 40), and do not experience acceleration along their trajectory. In this case, the beams of electron-positron plasma that are generated penetrate the stationary plasma of the Goldreich-Julian magnetosphere, which is composed of electrons and positively charged particles (probably, positrons), and is characterized by $\rho_{e f f}$. This should lead to the excitation of plasma oscillations both in the moving electron-positron plasma and in the stationary plasma, and, consequently, to the generation of radio emission. The corresponding radiation has two components: one represents a narrow beam, while the other is isotropic. The isotropic radio emission should have a lower characteristic frequency.

Let us determine which gamma rays can leave a tube through the lateral surface towards the region of the Goldreich-Julian plasma. With this aim in view, let us consider the variation of the line curvature with height.

In the model formulated above, we have the following expressions for the magnetic field $[9,14]$ :

$$
B_{r}=\frac{B_{0}}{\eta^{3}}, \quad B_{\theta}=\frac{B_{0}}{2 \eta^{3}}\left[\theta+2 \nu\left(\frac{\Delta \eta}{\eta-1+\Delta}\right)^{3}\right],
$$

where $\nu=B_{1} / B_{0}$. It can easily be shown that the radius of curvature of the field lines $\mathbf{B}$ can be expressed in terms of the function $f=B_{r} / B_{\theta}$ as

$$
\rho=r \frac{\left(1+f^{2}\right)^{3 / 2}}{1+f^{2}-\frac{d f}{d \theta}}
$$

We obtain for a dipolar field $\rho_{d i p}=\frac{4}{3} \frac{r}{\theta}$.

For a nondipolar field of the kind specified above, the curvature at $1<\eta<2$ is

$$
\rho=\frac{2 r}{3 \nu}\left(\frac{\eta-1+\Delta}{\Delta \eta}\right)^{3} .
$$

The following radii of curvature are obtained in this model: $\rho=1.1 \times 10^{6} \mathrm{~cm}$ at $\eta=1, \rho=10.4 \times 10^{6} \mathrm{~cm}$ at $\eta=1.2$, and $\rho=10^{8} \mathrm{~cm}$ at $\eta=2$. Therefore, the characteristic path of a photon leaving the tube can be estimated as $L>2.5 \times 10^{5} \mathrm{~cm}$.

For a gamma ray to leave the tube, it must not have a very large energy; i.e., it must be emitted by an electron in the initial stage of its acceleration. The energy of the gamma ray should satisfy the following inequality (for this estimation, we shall use here the path length obtained for the case of constant curvature):

$$
S_{p h}=\frac{5 \times 10^{12} \rho_{6}}{B_{12} \varpi \hbar}>L=2.5 \times 10^{5} .
$$


We find from this expression

$$
\varpi \hbar<\frac{2 \times 10^{7}}{B_{12}} \mathrm{eV}
$$

where $\rho_{6}=\rho / 10^{6}$.

Photons produced by the resonant inverse Compton effect will satisfy this inequality if

$$
\frac{B_{12}^{3}}{T_{5}}<\rho_{6} \approx 10
$$

In this case, the resonant photons will produce electron-positron pairs in the region of closed field lines.

Let us calculate the death line of a radio pulsar for the case of magnetic curvature radiation. The free path of a photon is

$$
S_{p h}=\frac{2 \times 10^{23} \rho_{6} \rho_{6}^{\prime}}{B_{12}^{\prime} \gamma^{3}}
$$

(primed and unprimed quantities refer to the points where the pair and gamma ray are produced, respectively).

The potential in a bent tube essentially reaches its maximum at a height of about $\eta=1.2$. Therefore, we take $\rho_{6}=10$, which corresponds to a height of $\eta=1.2$ and $\rho_{6}^{\prime}=50$.

Although the condition for pair formation $S_{p h}=$ $10^{6} \eta / 2$ was obtained for the case of constant curvature of the tube, we shall use this condition to estimate the death line for the case when the curvature changes with height.

This yields the death line $P=0.7 B_{12}^{2 / 3}$. If gamma rays from the tail of the spectrum $\left(E=10 E_{0}\right)$ are taken into account, $P=B_{12}^{2 / 3}$. If the stationary plasma is taken into account, $P=0.5 B_{12}^{2 / 3}$.

The death line for the inverse Compton effect is determined in a similar way:

$$
\frac{5 \times 10^{12} \rho_{6}^{\prime}}{B_{12}^{\prime} m c^{2} \gamma}=\frac{\eta}{2} 10^{6},
$$

and from this expression we obtain

$$
P=32 B_{12} \text {. }
$$

If we include the effect of filling of the unfavorable field lines with plasma, then

$$
P=18 B_{12} \text {. }
$$

The death lines for an asymmetric magnetic field with a favorable configuration are drawn in Fig. 4.

In the case under consideration, the death line extends far to the right. When the tube axis is bent unfavorably, the potential will decrease, and the death line will be shifted to the left compared to the dipolar

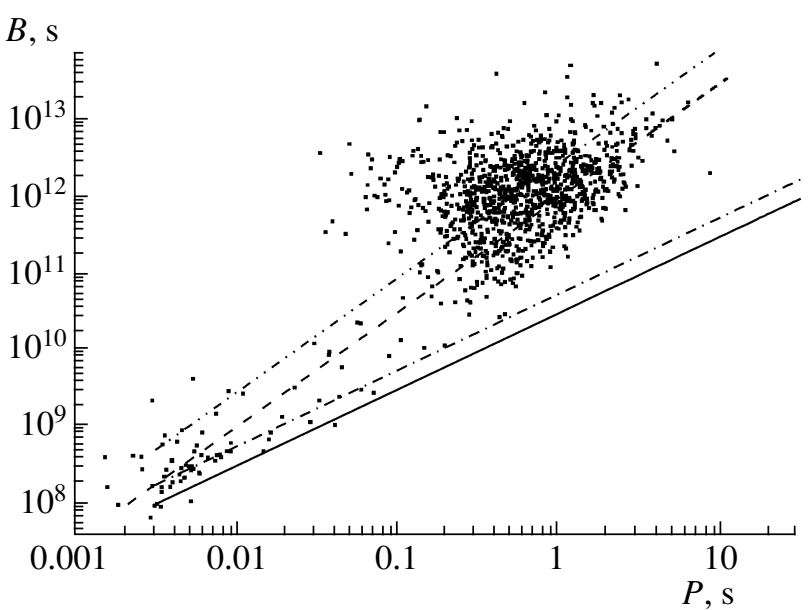

Fig. 4. Same as in Fig. 2 for the magnetic curvature mechanism (dashed), the inverse Compton effect acting on thermal photons from the entire stellar surface with stationary plasma (dash-dot), the inverse Compton effect acting on thermal photons from the entire stellar surface without stationary plasma (solid), and the magnetic curvature mechanism with stationary plasma (dash-doubledot).

case. In addition, it is possible that the run of the potential is such that the tube is filled with stationary plasma in the entire tube cross-section up to some height. In other words, the nondipolar magnetic field can also result in an earlier death of the radio pulsar.

Therefore, a nondipolar magnetic field in radio pulsars can lead to either an increase or decrease in the death periods.

\section{RESULTS}

We have obtained the death lines for the cases of dipolar and asymmetric magnetic fields, and estimated the multiplication factors for the secondary plasma in the case of a dipolar field.

If we assume that the pulsar radio emission is generated at the local frequency of Langmuir oscillations in the secondary-plasma beam, this frequency can be estimated as

$$
\varpi=1.4 \times 10^{10} \sqrt{k \gamma \frac{B_{12}}{P \eta^{3}}} \mathrm{rad} / \mathrm{s},
$$

where $\gamma$ is the Lorentz factor of the secondary plasma and $\eta$ is the normalized distance from the star to the spot where the oscillations are excited. This frequency turns out to be in the observable range for the multiplication factors obtained here.

If the field is dipolar and gamma rays are produced by the inverse Compton effect, the multiplication factor increases near the death line; i.e., the generation 
of secondary plasma becomes more efficient. As a result, more efficient radio emission by pulsars should be expected near the death line. In the case of an asymmetric magnetic field, the generation of radio emission is possible beyond the tube of open field lines.

\section{ACKNOWLEDGMENTS}

This work was supported by the Russian Foundation for Basic Research (project no. 04-02-17590), the Federal Scientific Technical Program Astronomiya (project 40.002.1.1.1103), and the Foundation for Noncommercial Programs Dinastiya.

\section{REFERENCES}

1. M. A. Ruderman and P. G. Sutherland, Astrophys. J. 196, 51 (1975).

2. R. N. Manchester and J. H. Taylor, Pulsars (Freeman, San Francisco, 1977; Mir, Moscow, 1980).

3. A. G. Muslimov and A. K. Harding, Astrophys. J. 568, $862(2002)$.

4. A. I. Tsygan and Kh. Spruit, Pis'ma Astron. Zh. 21, 877 (1995) [Astron. Lett. 21, 790 (1995)].
5. A. N. Muslimov and A. I. Tsygan, Astron. Zh. 67, 263 (1990) [Sov. Astron. 34, 133 (1990)].

6. A. G. Muslimov and A. I. Tsygan, Mon. Not. R. Astron. Soc. 255, 61 (1992).

7. V. S. Beskin, Pis'ma Astron. Zh. 16, 665 (1990) [Sov. Astron. Lett. 16, 286 (1990)].

8. A. I. Tsygan, Pulsar Astronomy-2000 and Beyond, Ed. by M. Kramer, N. Wex, and R. Wielebinski, ASP Conf. Ser. 202, 473 (2000).

9. E. M. Kantor and A. I. Tsygan, Astron. Zh. 80, 665 (2003) [Astron. Rep. 47, 613 (2003)].

10. T. Erber, Rev. Mod. Phys. 38, 626 (1966).

11. A. E. Shabat and V. V. Usov, Astrophys. Space Sci. 128, 377 (1986).

12. N. S. Kardashev, I. G. Mitrofanov, and I. D. Novikov, Astron. Zh. 61, 1113 (1984) [Sov. Astron. 28, 651 (1984)].

13. E. Tademaru, Astrophys. J. 183, 625 (1973).

14. V. D. Pal'shin and A. I. Tsygan, Preprint No. 1718 (Ioffe Phys.-Tech. Inst., 1998).

15. J. Arons and E. T. Scharlemann, Astrophys. J. 231, 854 (1979).

Translated by Yu. Dumin 